\title{
Congenital malrotation as an incidental finding after blunt trauma
}

\author{
L Ray Matthews, Omar K Danner \\ Travelyn Walker, Kenneth L Wilson
}

\begin{abstract}
Introduction: Blunt traumatic injuries can include polytrauma with extensive soft tissue injuries. Motor vehicle collision, motor cycle crashes, falls, assault, and pedestrians struck by automobiles are merely a few of several mechanisms by which trauma patients arrive in the emergency room. Case Report: During a systematic evaluation of a 50-year-old patient after a pedestrian versus automobile accident, head, chest, abdominal and pelvic computerized tomogram were obtained to evaluate her abdominal tenderness. Findings typically identified include injuries involving bone fractures, intracranial hemorrhage, intra thoracic and abdominal organ injuries. Incidental finding of congenital intestinal malrotation was seen on CT scans. Conclusion: We present an incidental finding of congenital intestinal malrotation in an adult patient after blunt force trauma.
\end{abstract}

Keywords: Malrotation, Trauma, Incidental findings

$* * * * * * * *$

L. Ray Matthews ${ }^{1}$, Travelyan Walker ${ }^{1}$, Kenneth L. Wilson ${ }^{1}$, Omar K. Danner ${ }^{1}$

Affiliations: ${ }^{1} \mathrm{MD}$ Morehouse School of Medicine, 720 Westview Drive, SW, Atlanta, GA 30310, USA.

Corresponding Author: L Ray Matthews, Morehouse School of Medicine, Department of Surgery, 720 Westview Drive, SW, Atlanta, GA 30310, USA; Ph: (404) 616-1415; Fax: (404) 616-1417; Email: lematthews@msm.edu

Received: 18 March 2011

Accepted: 17 August 2011

Published: 01 September 2011
Matthews LR, Danner OK, Walker T, Wilson KL. Congenital malrotation as an incidental finding after blunt trauma. International Journal of Case Reports and Images 2011;2(9):13-15.

$* * * * * * * * *$

doi:10.5348/ijcri-2011-09-54-CR-4

\section{INTRODUCTION}

Blunt traumatic injuries can include polytrauma with extensive soft tissue injuries. Motor vehicle collision, motor cycle crashes, falls, assault, and pedestrians struck by automobiles are merely a few of several mechanisms by which trauma patients arrive in the emergency room. The evaluation of these patients typically includes X-rays and Computed tomography (CA) scans to assess for occult injuries. Incidental findings are occasionally seen on CT scans. We present an incidental finding of congenital intestinal malrotation in an adult patient discovered after blunt force trauma. Malrotation usually presents in infants with abdominal wall defects and intestinal atresia, but occurs in association with Hirschsprung's disease, gastroesophageal reflux disease (GERD), intussception or other congenital malformations [1]. Our patient was completely asymptomatic, and if it were not for computed tomography (CT) scan of her abdomen and pelvis, we would have been completely unaware of her anomaly.

\section{CASE REPORT}

A 50-year-old female without any significant past medical or surgical history presented to the emergency room after being involved in a pedestrian versus automobile accident. The physical exam was unremarkable. CT scans of her abdomen revealed that 
the cecum and ascending colon (Figure 1, arrow) were located predominately on the left side. Also, the superior mesenteric vein and the superior mesenteric artery were inverted in respect to their normal anatomic relationship with the superior mesenteric vein being located to the left of the superior mesenteric artery instead of to the right as in their normal anatomic configuration (Figure 2, arrow) which is a diagnostic of congenital malrotation. She was discharged after 24-hour observation and remained asymptomatic.

\section{DISCUSSION}

During prenatal development of the abdominal cavity, the midgut must undergo proper rotation to prevent the bowel from twisting on its vertical axis. These staged maneuvers occur during gestational development and usually present early in infancy if development does not follow its appropriate course. In the fourth to sixth week of gestational development, the abdominal cavity is unable to accommodate the quickly growing viscera and the midgut prolapses through the umbilical ring and into the umbilical cord. By the tenth to twelfth week of gestation, the midgut returns to the abdominal cavity and rotates 270 degree in a counterclockwise direction around the vertical axis of the superior mesenteric artery. Ultimately, the cecum is positioned in the right lower quadrant and the third portion of the duodenum and ligament of Treitz in the left upper quadrant [2]. Deviation or reversal of these synchronized movements during embryologic development may lead to several midgut anatomical variations including non-rotation, incomplete rotation, or incomplete fixation [3]. The precise mechanism of intestinal malrotation is not well understood, although genetic mutations may predispose to malrotation. The incidence of intestinal malrotation is approximately 1 out of 500 live births and is usually seen in infants [4].

However, rotational anomalies may present as obstructive symptoms from midgut volvulus, chronic abdominal pain or as an incidental finding as seen in our patient necessitating close observation only. If obstruction or ischemia is present Ladd's procedure is a surgical option. Operative management for most symptomatic rotational anomalies includes Ladd's procedure. This procedure involves lysis of the adhesive bands to mobilize the midgut, counterclockwise reduction of the volvulus, widening of the midgut mesenteric pedicle, and appendectomy. Entrectomy is performed only for necrotic bowel which in the presence of ischemic bowel may require performing a temporary abdominal closure with reexploration in 24-36 hours [5].

\section{CONCLUSION}

Intestinal malrotation is a rare finding in adult

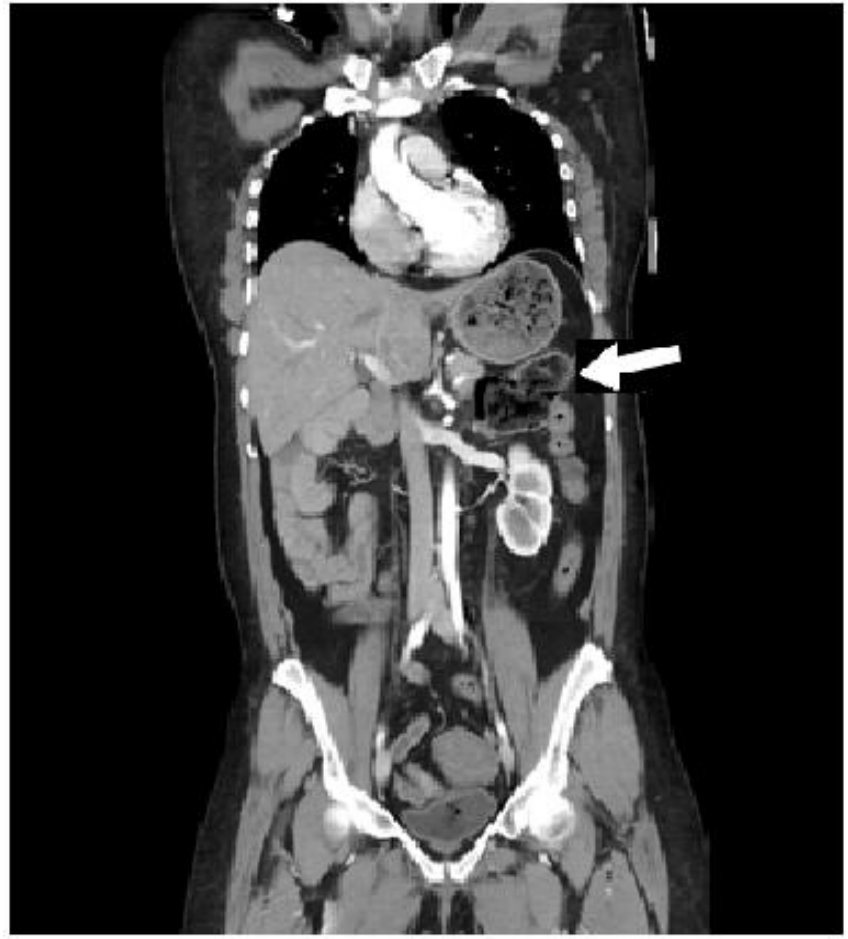

Figure 1: Abdominal CAT scan (coronal view) demonstrating the cecum and ileum malpositioned in the left upper quadrant in the usual location of the stomach.

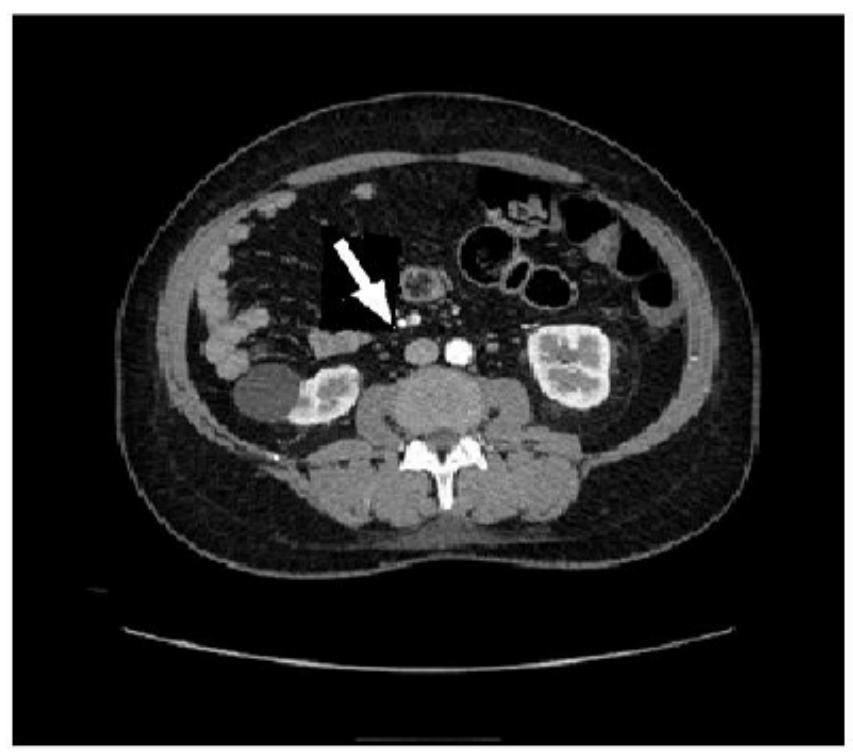

Figure 2: Abdominal CT scan demonstrating the inversion of the superior mesenteric artery and superior mesenteric vein.

patients and is usually found incidentally during radiographic imaging or surgical exploration for another disease process [6]. If the patient is symptomatic, further evaluation is needed to rule out bowel obstruction or mesenteric ischemia. 


\section{Author Contributions}

L. Ray Matthews - Substantial contributions to conception and design, Acquisition of data, Analysis and interpretation of data, Drafting the article, Revising it critically for important intellectual content, Final approval of the version to be published

Omar K. Danner - Substantial contributions to conception and design, Acquisition of data, Analysis and interpretation of data, Drafting the article, Revising it critically for important intellectual content, Final approval of the version to be published

Travelyn Walker - Substantial contributions to conception and design, Acquisition of data, Analysis and interpretation of data, Drafting the article, Revising it critically for important intellectual content, Final approval of the version to be published

Kenneth L. Wilson - Substantial contributions to conception and design, Acquisition of data, Analysis and interpretation of data, Drafting the article, Revising it critically for important intellectual content, Final approval of the version to be published

\section{Guarantor}

The corresponding author is the guarantor of submission.

\section{Conflict of Interest}

Authors declare no conflict of interest.

\section{Copyright}

(C) L. Ray Matthews et al. 2011; This article is distributed under the terms of Creative Commons attribution 3.0 License which permits unrestricted use, distribution and reproduction in any means provided the original authors and original publisher are properly credited. (Please see www.ijcasereportsandimages.com /copyright-policy.php for more information.)

\section{REFERENCES}

1. Kumaran N, Shankar KR, Lloyd DA, et al: Trends in the management and outcome of jejuno-ileal atresia. Eur J Pediatr Surg. 2002;12:163-7.

2. Kieswetter WB, Smith JW: Malrotation of the midgut in infancy and childhood. Arch Surg. 1958;77:483.

3. Dietz DW, Walsh RM, et al: Intestinal Malrotation. A rare but important cause of bowel obstruction in adults. Dis. Colon and Rectum. 2002;45(10):1381-6.

4. Malek M, Burd R: The optimal management of malrotation diagnosed after infancy: a decision analysis. Am J Surg. 2006;191:45-51.

5. Matzke GM, Dozois EJ, et al: Surgical management of intestinal malrotation in adults: comparative results for open and laparoscopic Ladd procedures. Surg Endosc. 2005;19:1416-9.

6. McVay M, Kokoska E, et al: The Changing spectrum of intestinal malrotation: diagnosis and management. Am J Surg. 2007;194:712-9. 